\title{
Los foros como estrategia de aprendizaje colaborativo los posgrados virtuales*
}

\author{
Ivonne López Cepeda \\ Tatiana Rosero Palacios ${ }^{* *}$
}

El objetivo de la investigación consiste en evaluar desde la experiencia de las maestrías de modalidad virtual, la forma en que se construye colaborativamente el conocimiento al interior de los grupos de aprendizaje utilizando los foros virtuales y las formas de relacionamiento entre pares y docentes como mediadoras de los procesos educativos.

En el estudio se trabajó con una muestra de diecisiete foros generales y grupales correspondientes a once asignaturas de los programas de maestría en Gestión Educativa, Política Social para la Infancia y la Adolescencia y Pastoral Juvenil, desarrolladas en metodología virtual. Se hace una descripción y análisis de la forma en que se construye colaborativamente el conocimiento al interior de estos grupos virtuales de aprendizaje, de sus formas de correspondencia y del nivel de participación que se genera como consecuencia de estas relaciones y compromisos, de las formas comunicativas de los participantes y del rol de mediador del docente desde su función como planificador, ejecutor y evaluador del proceso educativo.

Las experiencias educativas generadas en la UPS desde los programas de maestría en modalidad virtual o e-learning se fundamentan en el constructivismo sociocognitivo (Badia 2006), corriente que conjuga principios psicopedagógicos de las teorías cognitivas y sociales del aprendizaje. Esta definición pedagógica e instruccional ha sido adoptada en función de la concordancia con el modelo pedagógico salesiano basado en una pedagogía del acompañamiento y en una educación liberadora.

En la UPS, el desarrollo de la modalidad de estudios no presenciales mediados por entornos virtuales de aprendizaje (EVA) se da a través de una metodología fundamentada en cuatro ejes: socio-cultural, pedagógico, comunicacional y tecnológico. Para el desarrollo de los diferentes programas y cursos

\footnotetext{
* $\quad$ Este artículo es producto de la investigación "Los foros como estrategia de aprendizaje colaborativo. Evaluación de la experiencia de los posgrados virtuales en la Universidad Politécnica Salesiana”, financiada por la Universidad Politécnica Salesiana, UPS.

** Profesoras investigadoras de la Universidad Politécnica Salesiana.
} 
se ha conformado un equipo multidisciplinario, que atiende requerimientos de coordinación y asesoría para el diseño y planificación curricular, de formación y apoyo a la docencia virtual y a los grupos de estudiantes, de gestión del entorno virtual para la administración técnica de las aulas virtuales y el soporte a usuarios, de gestión administrativa que implica la atención al destinatario en la provisión de información actualizada, mediación para la comunicación del estudiante con diversas instancias de la UPS, proceso de inscripción, matrículas $\mathrm{y}$ el registro de notas.

Las experiencias educativas en el nivel de estudios de posgrado y las mediaciones dadas a través del entorno virtual en el que se desarrollan los aprendizajes son importantes referentes para la contextualización de nuestra propuesta de e-learning, por eso las relaciones entre estudiantes, y entre estos y el docente, se hacen fundamentales. El uso de foros constituye así la principal estrategia de aprendizaje colaborativo en el EVA, en la cual intervienen algunas consideraciones teóricas respecto a la construcción social del conocimiento, los principios del aprendizaje colaborativo, los niveles de interacción entre los participantes a través de los espacios de discusión y la determinación de criterios de evaluación del trabajo académico en los foros.

Este estudio -siguiendo la tendencia mayoritaria de evaluación de experiencias de e-learning- hace una revisión cuantitativa de las interacciones y dinámicas de los grupos para dimensionar el nivel de participación entre estudiantes y docentes. Aporta además en una línea menos trabajada como es la evaluación cualitativa de los procesos de aprendizaje colaborativo en medios virtuales desde un análisis del rol del estudiante como actor protagónico de su propio aprendizaje, análisis que se hace desde la percepción de la interactividad y de las formas de comunicación al interior de los grupos que les permite negociar o no los sentidos y definiciones de las categorías conceptuales que se discuten como contenido de los debates, para determinar si el proceso de aprendizaje colaborativo se cumple a través de un foro virtual.

\section{Marco Teórico}

\section{Ejes para el andamiaje del e-learning}

El desarrollo de la modalidad de estudios no presenciales mediados por entornos virtuales de aprendizaje (EVA) se da desde consideraciones particulares 
relativas a la trayectoria institucional, al programa educativo, a los destinatarios, al contexto, a la disciplina, etc. Respetando las especificidades de las instituciones y de sus ofertas académicas la metodología del e-learning ha de estar sostenida en cuatro grandes ejes: socio-cultural, pedagógico, comunicacional y tecnológico.

\subsection{Eje socio-cultural e institucional}

La inclusión de las tecnologías de la información y la comunicación (TIC) en los procesos educativos es un requerimiento contemporáneo para toda institución involucrada en este ámbito. Las referencias para contextualizar una oferta educativa de e-learning se vinculan con la filosofia institucional, su organización y capacidad para responder a las demandas, las perspectivas de formación de los destinatarios; con la propuesta y su diseño curricular, con los recursos tecnológicos, con las competencias digitales, con las competencias comunicacionales de los usuarios, etc.

Así la propuesta de mediaciones didácticas y tecnológicas que soporten la oferta educativa estará dada desde la consideración y conocimiento explícito del perfil del estudiante de manera que su formación y experiencias previas personales y laborales- sean el insumo que permite establecer estrategias para el diálogo y el trabajo en grupos según los objetivos de aprendizaje que se hayan formulado.

\subsection{Eje pedagógico}

La definición pedagógica e instruccional para las experiencias educativas generadas en modalidad virtual se adaptará al modelo pedagógico y de gestión de la institución educativa y, en términos amplios, debe estar fundamentada en los principios psicopedagógicos de las teorías cognitivas y sociales del aprendizaje correspondientes al constructivismo sociocognitivo (Badia 2006).

Desde estos planteamientos y opciones pedagógicas y metodológico-didácticas se hace un expreso reconocimiento al hecho de que:

...tanto la cognición individual del estudiante como la interacción social educativa que cada uno establece con el profesor y los compañeros deben considerarse, 
en gran medida, como procesos situados y claramente influidos por las características del contexto educativo donde tienen lugar. (Badia 2006, 6-7)

En concreto, cognición e interacción, son cualificadores explícitos de la propuesta de actividades o tareas de una asignatura. En toda tarea son indisociables cuatro componentes didácticos: el objetivo, la metodología para llegar al objetivo, los materiales de estudio y el porcentaje de evaluación de cada actividad dentro de la asignatura.

\subsection{Eje comunicacional}

En el EVA, los actores se interrelacionan durante todo el proceso de enseñanza-aprendizaje, prioritariamente mediante estrategias de comunicación asincrónica y en menor porcentaje de forma sincrónica permitiendo la flexibilización en los horarios de acceso a los espacios de encuentro; en esta propuesta, tanto docentes como estudiantes requieren del desarrollo de mayores destrezas en la comunicación escrita.

El proceso formativo es solo posible en cuanto haya comunicación, interacción y construcción colectiva del conocimiento. Para ello, es condición necesaria el manejo apropiado de la información, no solo para su búsqueda-selecciónprocesamiento-apropiación-aplicación, sino para resignificarla y convertirla en conocimiento.

El diseño de un entorno virtual se basa en los factores antes mencionados y en el manejo de espacios para la comunicación, de relacionamiento interpersonal a través de foros informales y servicio de mensajería; además de espacios de interacción y participación colectiva para la gestión del conocimiento a través de foros académicos generales, foros grupales y salas de chat. Además de apartados para la gestión de la información.

De este modo se conjugan propuestas y sistemas que armonizan la gestión del contenido y la gestión del proceso de enseñanza y de aprendizaje.

\subsection{Eje tecnológico}

Existen infinidad de ofertas o sistemas que permiten el diseño de entornos virtuales de aprendizaje, su selección y aplicación depende de las particularidades institucionales y de sus perspectivas respecto de la educación en línea. 
Una plataforma usual en nuestro medio (país y región) es MOODLE ${ }^{1}$ (Modular Object-Oriented Dynamic Learning Environment), software de licencia abierta que constituye para nuestros requerimientos y desde nuestros planteamientos todo un "Sistema de Gestión de Cursos de Código Abierto (Open Source Course Management System, CMS), conocido también como Sistema de Gestión del Aprendizaje (Learning Management System, LMS)"

A partir de las herramientas y recursos que ofrece el sistema se estructura el EVA. A continuación se ejemplifica la organización de la oferta virtual de la Universidad Politécnica Salesiana según el tipo de programa, la edición correspondiente y según el número de grupos que se hayan abierto a partir de la demanda.

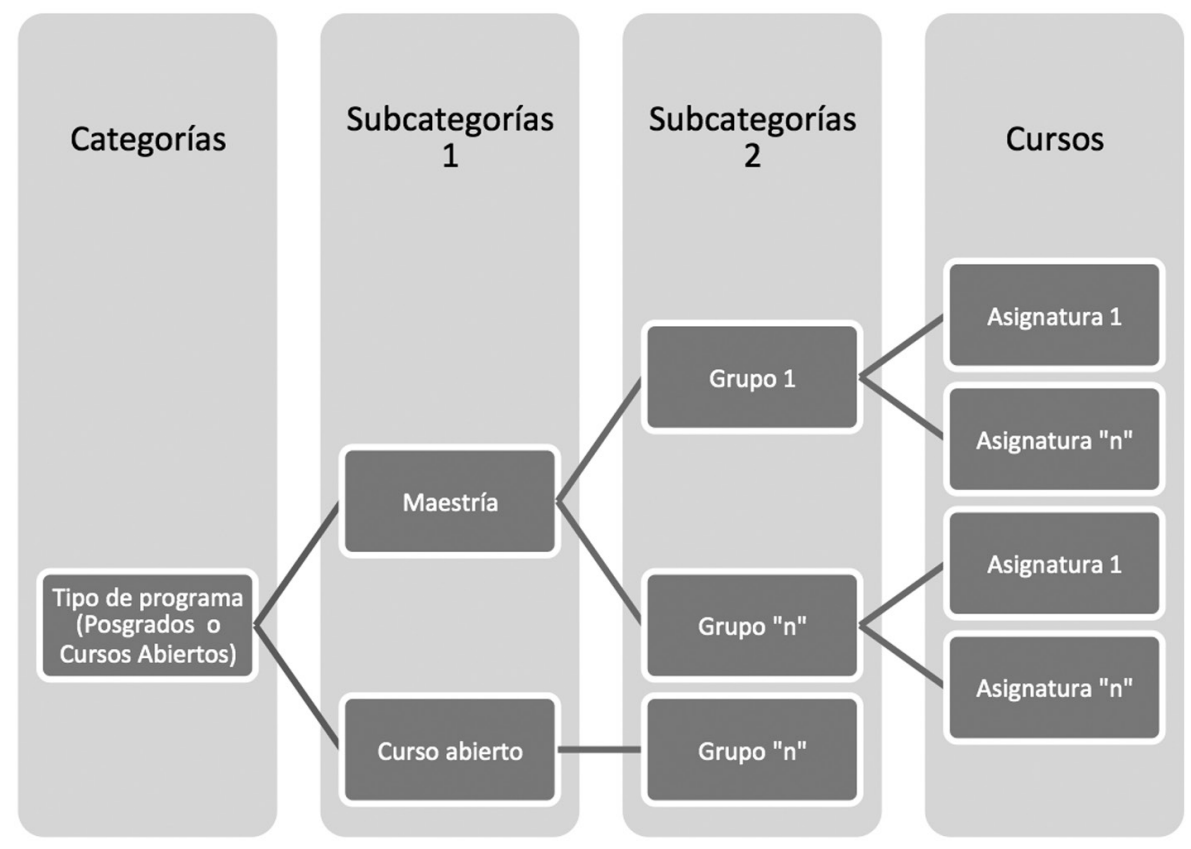

Se sugiere revisar: http://moodle.org/ 


\section{Comunidad virtual de aprendizaje y entorno virtual de aprendizaje}

El entorno virtual de aprendizaje es una aplicación informática desarrollada para Internet que tiene su base en el concepto de Comunidad Virtual (CV). Una CV se entiende como la convergencia de personas inmersas en un entorno donde pueden comunicarse, intercambiar información e identificarse con otros miembros por similitud de intereses o posturas respecto de ciertas temáticas. Las Comunidades Virtuales han surgido desde distintas finalidades sociales, culturales o económicas, no reemplazan las articulaciones sociales en la cotidianidad, sino que son parte de ellas, son un ámbito diverso y adicional de socialización.

La participación en una CV está marcada por los objetivos e intereses que determinan y comparten los miembros de esa comunidad virtual; César Coll, Alfonso Bustos y Anna Engel ${ }^{2}$ proponen la existencia de "comunidades virtuales de interés" (CVI), "comunidades virtuales de participación" (CVP) y "comunidades virtuales de aprendizaje" (CVA). La dinámica grupal al interior de cada uno de estos tipos de comunidad puede transformarla en un tipo distinto, como en muchos casos las clasificaciones tienen límites muy sensibles cuando se trata de interrelaciones sociales, sin embargo, esta tipología resulta útil para enfatizar que la educación formal a través de entornos virtuales -o comunidad virtual de aprendizaje- ha de desarrollarse desde un diseño instruccional pedagógico pensado desde el qué, el cómo, el quién, el dónde, el con qué y el para qué del proceso educativo.

La CVA difiere de cualquier otro tipo de iniciativa libremente conformada en Internet, pues la suscripción y acceso a dicha comunidad está determinada tanto por la institucionalidad del centro educativo que administra el espacio virtual, como por la predisposición de cada participante a generar desde allí sus procesos de aprendizaje.

Otra cualidad que garantiza el uso justificado de un EVA es la existencia -en la institución educativa- de un equipo multidisciplinar que gestiona los procesos de enseñanza y de aprendizaje, además es el que da soporte a todos los

2 Ampliar información en Las comunidades virtuales de aprendizaje, apartado escrito por los autores para el capítulo XIII del libro Psicología de la educación virtual de Coll y Monereo, edición 2008. 
usuarios: equipo docente, estudiantes, coordinación académica del programa, contenidistas.

Desde este diseño instruccional se hace la mediación pedagógica que tiene como insumo fundamental la planeación curricular. La propuesta de actividades está en línea directa de relación con los objetivos de aprendizaje, con el contexto disciplinar y laboral o de aplicación del conocimiento, con los criterios de evaluación.

La salvedad que vale la pena señalar, es que las nuevas tecnologías aplicadas a la educación, especialmente Internet, ofrecen "realismo" y no "realidad", esto significa que queda pendiente un imperativo ético como responsabilidad de la persona para ensamblar el andamiaje de la información y el conocimiento con las circunstancias históricas; de hecho lo mismo ocurre con los aprendizajes tradicionales: se corre el riesgo de que se queden en teoría, en las aulas, en las bibliotecas y en los laboratorios (Picardo Joao 2002).

Esta cita tomada de Picardo, nos introduce a la importancia de la planificación de los procesos educativos a nivel micro curricular en cuanto a previsión de actividades significativas y recursos requeridos para el aprendizaje. Al referirnos a lo curricular, encontramos que coexisten variados enfoques y definiciones de currículo, sin embargo, nos acogemos a la siguiente:

...currículum es el eslabón entre la cultura y la sociedad exterior a la escuela y la educación, entre el conocimiento o la cultura heredados y el aprendizaje de los alumnos, entre la teoría (ideas, supuestos y aspiraciones y práctica posible, dadas unas determinadas condiciones... el currículum es la expresión y concreción del plan cultural que una institución escolar hace realidad dentro de unas determinadas condiciones que matizan ese proyecto, (Gimeno 1988 citado en Córica 2009; 88).

Siendo el currículo un vínculo entre el contexto social y el contexto educativo, el plan microcurricular articulado con el entorno virtual se constituyen en un canal de comunicación de las intencionalidades y expectativas educativas tanto de los educadores como de los educandos; y también, en el espacio donde la experienciación hace efectivos dichos intereses. Es decir, el complejo sistema de relaciones entre el docente, el estudiante y el objeto de estudio se 
lleva a cabo en dicho entorno y su configuración está prevista en el plan microcurricular.

El entorno virtual configurado para el aprendizaje, entonces, proporciona dos experiencias fundamentales a la persona: interactividad e interacción social $^{3}$ (Coll, Mauri y Javier 2008; 37)

\subsection{Evaluación y juicios de valor ${ }^{4}$}

En la interacción se van construyendo juicios de valor sobre la participación del otro y simultáneamente se construyen juicios sobre sí mismo a partir del reconocimiento que los otros hacen de nosotros.

Respecto de la evaluación, expresada a través de juicios de valor y sin entrar en la discusión sobre sus relaciones de poder, es preciso tener presente que genera un conflicto entre la objetividad que argumenta considerar quien evalúa y la subjetividad que siente quien ha sido evaluado. Esta dicotomía, al decir de Mancovsky, surge de la arbitrariedad de lo que se evalúa en un hecho o su naturaleza, de sus funciones y de sus procesos de aplicación. La evaluación “...siempre va a estar referida a: normas de conducta y valores que dan cuenta de un modelo esperado en relación al trabajo escolar (el orden en el aula) o en relación con un modelo ideal de alumno ('sus logros en el aprendizaje')" (Mancovsky 2000; 95). Desde esta visión más que evaluar los aprendizajes se evalúa el cumplimiento del "trabajo escolar" (Mancokvsky 2000; 92) o las tareas o las actividades diseñadas por el docente en el plan curricular. Pero cumplir o no cumplir son factores de cualificación para el prestigio y reconocimiento académico del docente hacia el estudiante y de estos entre sí.

Entendida la evaluación como el proceso a través del cual un sujeto -generalmente el docente en el caso de la heteroevaluación- emite un juicio de valor sobre un objeto, procedimiento o hecho educativo, es necesario precisar que

3 ... los autores distinguen entre interacción, referida a la actividad de las personas, e interactividad, relacionada con las características tecnológicas del sistema. Basándose en esta distinción, los autores definen interacción social como el intercambio social que se produce entre individuos y grupos que se influencian unos a otros, enfatizando los aspectos comunicativos del intercambio de información (Coll, Mauri y Javier 2008; 37).

4 Los juicios de valor "tienen por objeto expresar, no aquello que las cosas son, sino lo que ellas valen en relación a un sujeto consciente, es decir, el precio a que éste último se ajusta”. "Todos los valores existen en un sentido dado, fuera del sujeto". (Durkheim, E., 1924, citado por Mancovsky 2000; 94). 
el desempeño de un estudiante y su cumplimiento de tareas va a ser retroalimentado a través de tres tipos de enunciados que pretenderán darle evidencias del avance o logro de los objetivos de aprendizaje definidos para la asignatura, tema o tarea específica. Desde los aportes de Viviana Mancovsky, se considera la evaluación a través de:

- Enunciados descriptivos: cuando hace referencia y cualifica hechos, actitudes individuales y colectivas.

- Enunciados persuasivos: cuando los mensajes expresan emociones y sentimientos personales con la intención de incidir en las emociones y modos de pensar de los otros.

- Enunciados prescriptivos: cuando la comunicación tiene como propósito guiar la acción de los participantes.

\section{La interacción y la comunicación como factores de cons- trucción colectiva para la gestión del conocimiento}

A partir de la inclusión de las TIC y de Internet en los procesos de enseñanza-aprendizaje en las ofertas educativas no presenciales, aparece una serie de propuestas para potenciar el relacionamiento entre los sujetos y el objeto de estudio, a través de distintas metodologías y estrategias didácticas para la aplicación de la diversidad de formatos de presentación del contenido y de variedad de herramientas que permiten la comunicación.

De esta manera, se pueden identificar tres tipos posibles de interacción en el EVA, considerando la interrelación entre los sujetos (docentes y estudiantes) y con el objeto de estudio como el paso de una concepción de actividad individual hacia un "proceso constructivo de naturaleza interactiva, social y cultural" (Monereo, Carles y Pozo en Coll \& Monereo 2008; 140).

Las interacciones identificadas son: a) Interacción entre docentes y estudiantes, b) Interacción entre los estudiantes y los contenidos, y c) interacción entre el docente y los contenidos.

La interacción docente-contenidos, está vinculada al conocimiento experto, es decir a la mediación que el docente realiza respecto de los sentidos y significaciones producidas a través de la negociación social en cada ámbito científico o disciplina, proviene del reconocimiento de las "voces autorizadas" 
del contexto social-cultural, del posicionamiento institucional y docente frente al estado del arte del objeto de estudio.

Lo referido a la interacción estudiante-objeto de estudio, corresponde a la autonomía del estudiante en la elaboración y resignificación del contenido y es producto del la interrelación directa con los materiales propuestos en el EVA $\mathrm{y}$ que puede ser alimentado por fuentes de medios externos al aula virtual, a través de la red o desde la información que toma y confronta desde el entorno cercano del estudiante.

Para el análisis de los foros como estrategia de aprendizaje, esta investigación profundiza el estudio alrededor de la interacción entre los sujetos y con la tecnología como elemento fundamental de la construcción social y la gestión del conocimiento en los EVA. También, se determina dos tipos de actividades comunes de utilización del foro que son la discusión didáctica y el trabajo colaborativo.

\subsection{Fases de la construcción social del conocimiento de los foros en $\boldsymbol{E} \boldsymbol{V A}$}

Son varios los autores que han investigado y analizado la dinámica de los foros de discusión y el trabajo colaborativo en los EVA, de los cuales se pueden destacar los modelos citados en (Coll y Monereo 2008; 243-245), a saber: el de análisis de calidad de los mensajes propuesto por Henri (1992) y el análisis de una comunidad de indagación de Garrison y Anderson (2005). Así también, se considera el aporte de López y Álvarez (2009; 166) a través del análisis de los modos de regulación durante la actividad cooperativa. 
Tabla relacional de enfoques sobre la dinámica en foros de discusión y trabajo colaborativo

\begin{tabular}{|c|c|c|c|}
\hline $\begin{array}{l}\text { Dimensiones de } \\
\text { análisis sobre } \\
\text { calidad de los } \\
\text { mensajes (Henri) }\end{array}$ & $\begin{array}{c}\text { Fases y grado de } \\
\text { "presencia cognitiva" } \\
\text { (Garrison y Anderson) }\end{array}$ & $\begin{array}{c}\text { Grado de "presencia } \\
\text { social"s (Garrison y } \\
\text { Anderson) }\end{array}$ & $\begin{array}{c}\text { Fases de la actividad } \\
\text { cooperativa (López \& } \\
\text { Álvarez) }\end{array}$ \\
\hline $\begin{array}{l}\text { Participativa } \\
\text { Número de cada } \\
\text { estudiante durante } \\
\text { el diálogo. }\end{array}$ & $\begin{array}{l}\text { De iniciación } \\
\text { Abordaje inicial del } \\
\text { tema; exposición breve } \\
\text { de las ideas principales } \\
\text { a desarrollar. } \\
\text { Bajo nivel de } \\
\text { complejidad. }\end{array}$ & $\begin{array}{l}\text { Comunicación abierta } \\
\text { Mensajes de saludo } \\
\text { y/o presentación; de } \\
\text { coordinación de una } \\
\text { actividad específica. }\end{array}$ & $\begin{array}{l}\text { Inicio Estrategias } \\
\text { reguladoras sociales } \\
\text { para el análisis de los } \\
\text { requerimientos de la } \\
\text { tarea y los términos de la } \\
\text { cooperación. }\end{array}$ \\
\hline $\begin{array}{l}\text { Interactiva } \\
\text { Interconexión } \\
\text { y referencias } \\
\text { mutuas entre los } \\
\text { participantes. }\end{array}$ & $\begin{array}{l}\text { De exploración } \\
\text { Compartir y comparar } \\
\text { ideas e información. } \\
\text { Mediana complejidad. }\end{array}$ & $\begin{array}{l}\text { Cooperación y liderazgo } \\
\text { Mensajes para coordinar el } \\
\text { desarrollo de una actividad } \\
\text { específica. } \\
\text { Iniciativa de liderazgo en el } \\
\text { grupo. }\end{array}$ & $\begin{array}{l}\text { Intercambio } \\
\text { Corregulación del } \\
\text { comportamiento y del } \\
\text { discurso propio de cada } \\
\text { integrante (discurso } \\
\text { propio). }\end{array}$ \\
\hline $\begin{array}{l}\text { Cognitiva } \\
\text { Tipo de } \\
\text { procesamiento y } \\
\text { comprensión de la } \\
\text { información. }\end{array}$ & $\begin{array}{l}\text { De integración } \\
\text { Debate de conceptos } \\
\text { o afirmaciones y } \\
\text { negociación de } \\
\text { significado. Alta } \\
\text { complejidad. }\end{array}$ & $\begin{array}{l}\text { Comunicación afectiva y } \\
\text { cohesiva } \\
\text { "capacidad para proyectarse } \\
\text { social y emocionalmente } \\
\text { como personas reales" } \\
\text { Reconocimiento de la } \\
\text { autoridad de quien puede } \\
\text { liderar y representar al grupo } \\
\text { frente al docente. } \\
\text { Mensajes de motivación y } \\
\text { conducción de la línea de } \\
\text { discusión. }\end{array}$ & $\begin{array}{l}\text { Negociación } \\
\text { Corregulación para } \\
\text { la elaboración flujo } \\
\text { de razonamientos } \\
\text { argumentados y } \\
\text { construcción conjunta de } \\
\text { conocimiento. }\end{array}$ \\
\hline $\begin{array}{l}\text { Metacognitiva } \\
\text { Comprensión de la } \\
\text { dinámica y de las } \\
\text { mejores maneras } \\
\text { para llegar a } \\
\text { construir un } \\
\text { discurso conjunto. } \\
\text { Sistematización } \\
\text { individual o } \\
\text { colectiva de los } \\
\text { aportes. }\end{array}$ & $\begin{array}{l}\text { De resolución } \\
\text { Construcción } \\
\text { colaborativa de ideas } \\
\text { nuevas. Elaboración del } \\
\text { discurso colectivo. } \\
\text { Alto nivel de } \\
\text { complejidad. }\end{array}$ & $\begin{array}{l}\text { Comunicación cohesiva y } \\
\text { afectiva } \\
\text { Mensajes de cierre de la } \\
\text { discusión, indicando que } \\
\text { se ha llegado a un acuerdo } \\
\text { o a un nivel máximo de } \\
\text { construcción del discurso } \\
\text { colectivo. }\end{array}$ & $\begin{array}{l}\text { Aplicación } \\
\text { Estrategias reguladoras } \\
\text { sociales para presentación } \\
\text { del producto final. }\end{array}$ \\
\hline
\end{tabular}

5 La aproximación de Henri (1992) incluye la dimensión social referida a mensajes individuales que no están relacionados directamente con la tarea, sin embargo no es suficiente para explicar el proceso de construcción social del conocimiento. 
En el siguiente gráfico se expresa la conjunción de lo propuesto por los autores antes citados identificando las etapas de la dinámica en los foros de discusión y de trabajo colaborativo.

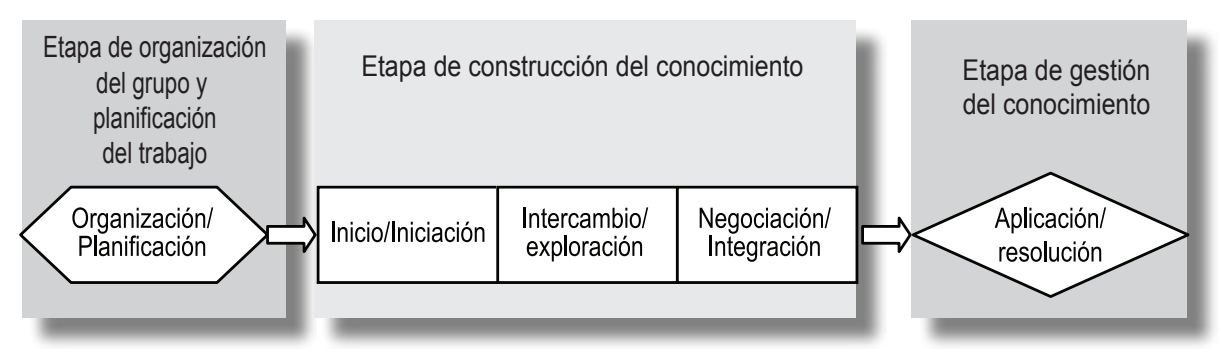

Se debe apuntar que el gráfico indica un proceso ideal de interacción en EVA, en el cual la discusión se desarrolla de manera totalmente lineal, no se ha incluido el carácter hipertextual y asincrónico de la herramienta.

Por esta razón, es necesario analizar también los elementos que permiten reconocer el tipo de mensaje que se ha producido, para determinar el momento del proceso en el cual se inscriben; para esto, se toman en cuenta las categorías definidas por Kerbrat-Orecchioni (1990) citado en (Mancovsky 2000; 101):

- Alocución, momento que indica el reconocimiento de un destinatario de la comunicación. Es decir, la presencia de un locutor y un "otro" diferente al locutor.

- Interlocución, espacio en que se da el intercambio y circulación de mensajes, así como el intercambio de los papeles de quien emite o recibe la información (emisión-respuesta). Esto define la presencia de interlocutores.

- Interacción, donde los interlocutores establecen un diálogo fluido y se "hablan" alternativamente constituyéndose en "una actividad colectiva de producción de sentido". 


\subsection{La comunicación escrita como elemento fundamental en la interacción}

Es necesario, asimismo, para el análisis de los mensajes incluir elementos de la comunicación visual y específicamente la comunicación escrita, donde el lenguaje es la expresión de los sentidos y significados que se van elaborando individual y colectivamente.

En la elaboración de los mensajes intervienen el nivel de complejidad del lenguaje especializado y/o específico de la disciplina o ámbito de estudio (metalenguaje), las estructuras sintácticas, la extensión y profundidad con la que se han desarrollado las ideas, así como el uso de recursos gráficos como son diferencia de tipo de letra (forma o color) o elementos iconográficos (por ejemplo, emoticones). El análisis de todos estos elementos en conjunto, más la consideración del perfil o caracterización del grupo en que se da la interrelación en el foro de discusión, dan cuenta de la "lecturabilidad" o grado de dificultad que puede presentar el texto del mensaje para su comprensión por parte de todos los participantes ${ }^{6}$.

La correlación de estos elementos, también nos indica la articulación del mensaje en relación a las diferentes líneas de discusión que se puedan generar, ya sea desde mediación docente o desde el interés particular de los estudiantes.

A partir de la mediación del docente, cuya primera evidencia está en el enunciado de la actividad y el mensaje de inicio o apertura del foro, podemos establecer tres tipos de estrategia orientados según el objetivo de aprendizaje, y son foros de pregunta/respuesta, de discusión didáctica y trabajo grupal. Los dos primeros tipos suelen estar dirigidos a la verificación del dominio de teorías, principios, conceptos y categorías, a través del control de lectura o confrontación argumentada de posturas; mientras que, el tercer tipo suele estar vinculado a actividades de producción colaborativa de integración de objetivos de aprendizaje. (Medina Moya 2006; Medina y Salvador 2002)

6 Si bien en el concepto de "lecturabilidad" en su inicio se adoptó como sistema de medición cuantitativa para determinar la legibilidad o facilidad de comprensión del texto (IICA-CIRA, 1973:I-D-2 y 3), en la actualidad se articula con variables cuantitativas (como número de palabras o signos utilizados) a elementos cualitativos para obtener resultados integrales de análisis del sentido (Montesi 2009; 98-99). 
Los estilos y formas expresivas utilizadas para la negociación de sentidos en un debate son diversos y pueden construirse desde dos líneas discursivas inherentes a la educación:

- Desde el discurso educativo o discurso didáctico -como lo denomina Coll para hacer referencia a un tratamiento más amplio del manejo comunicativo en las experiencias educativas-, en el que se inscriben estrategias discursivas de tipo informativo como son la narración, la descripción, la exposición, la argumentación, la explicación.

- Desde el discurso cotidiano, que al decir de Daniel Prieto Castillo sus mensajes hacen referencia a la inmediatez de las relaciones, al bajo nivel de análisis de un objeto o acontecimiento, a la agilidad para hacer clasificaciones o tipificaciones para reconocer algo rápidamente, a la organización de las rutinas cotidianas, al juego abierto o sutil del autoritarismo y el ejercicio del poder, al reconocimiento social que se logra a través del discurso. En este estudio se hace una aplicación de las características del discurso cotidiano al tratamiento de temas académicos, así se definen los indicadores: mensajes coloquiales con rigor académico y mensajes coloquiales sin rigor académico.

\section{Metodología}

La investigación es de tipo descriptivo y analítico de los elementos cualitativos y cuantitativos seleccionados para evaluar los logros de la metodología del foro virtual como estrategia didáctica para los procesos formativos de los programas de posgrado que se ofrecen en modalidad virtual en la UPS.

\section{Muestra}

La muestra consta de 17 foros: 10 foros generales y 7 foros grupales, correspondientes a 11 asignaturas: 3 de la Maestría en Educación mención Gestión Educativa, 3 de la Maestría en Pastoral Juvenil y 5 de la Maestría en Política Social de la Infancia y Adolescencia. 


\begin{tabular}{|c|c|c|c|c|}
\hline 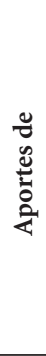 & 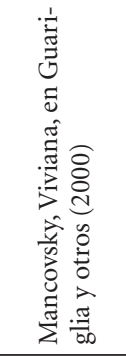 & 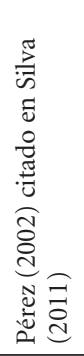 & 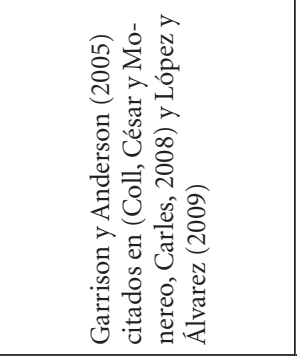 & 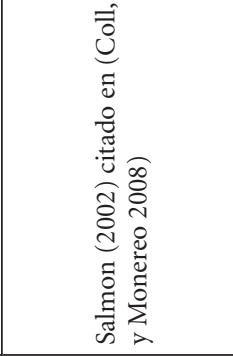 \\
\hline 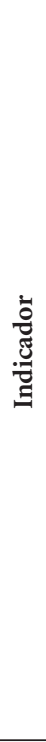 & 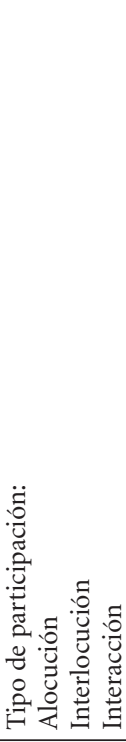 & 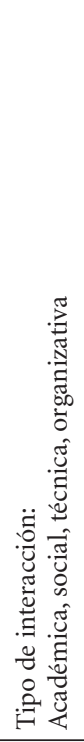 & 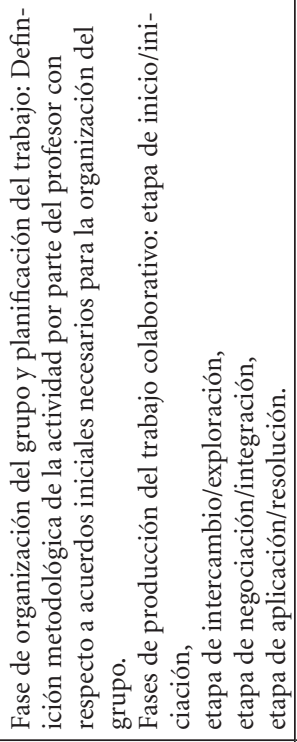 & 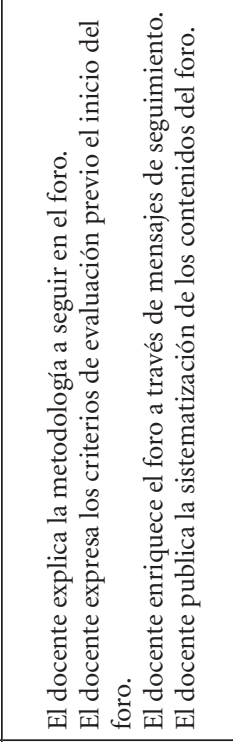 \\
\hline 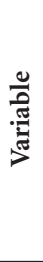 & 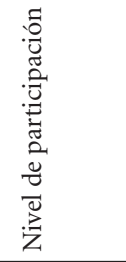 & 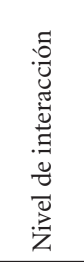 & 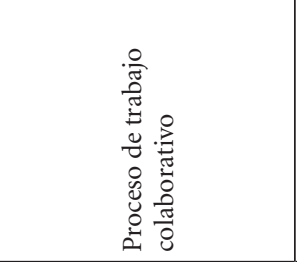 & 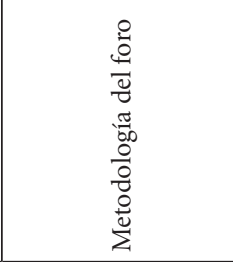 \\
\hline$\frac{2}{2}$ & \multicolumn{3}{|c|}{ 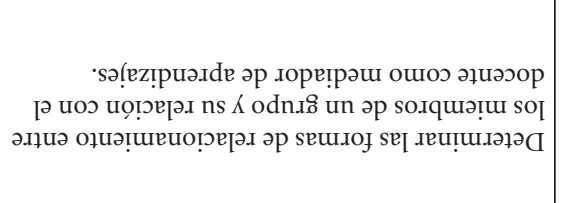 } & 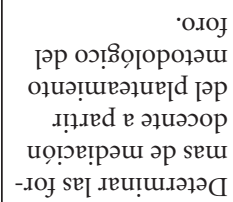 \\
\hline
\end{tabular}




\begin{tabular}{|c|c|c|c|c|}
\hline 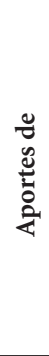 & & 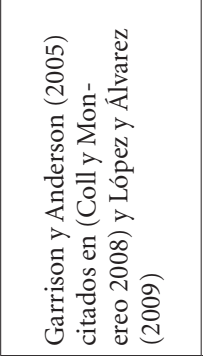 & 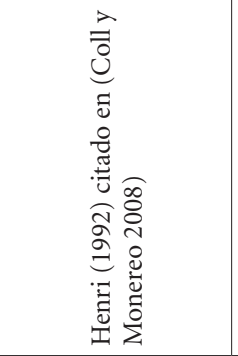 & 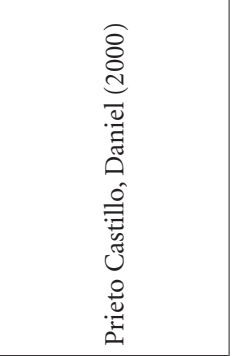 \\
\hline 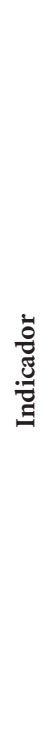 & 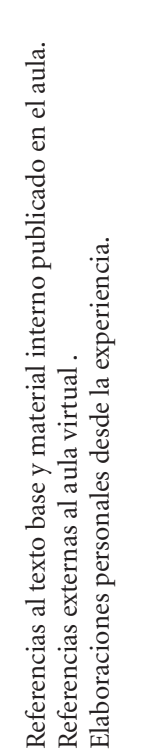 & 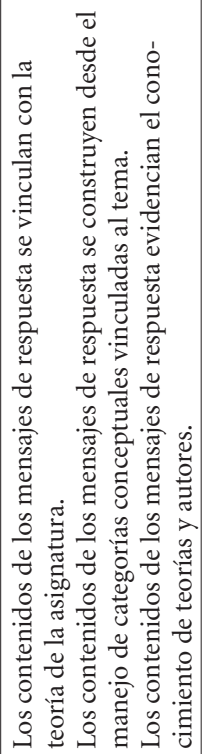 & 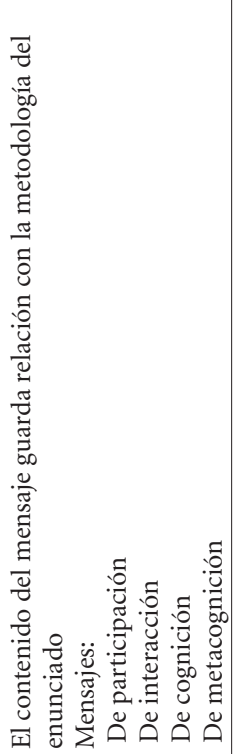 & 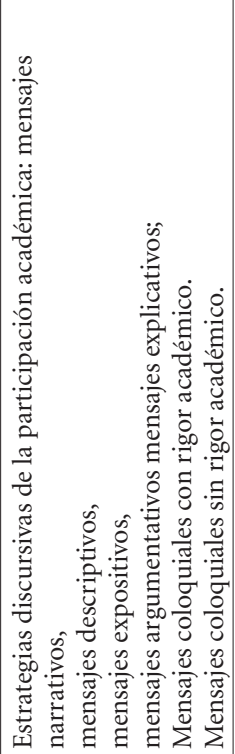 \\
\hline $\begin{array}{l}\frac{0}{0} \\
\frac{\pi}{\tilde{n}} \\
\frac{\pi}{\nu}\end{array}$ & 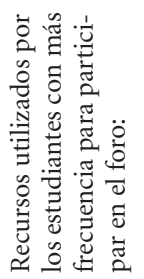 & 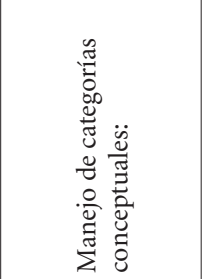 & 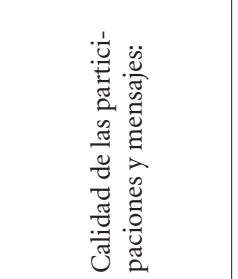 & 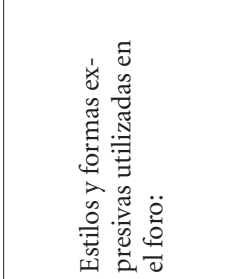 \\
\hline $\begin{array}{l}\frac{Z}{0} \\
: 0 \\
0\end{array}$ & әұนеب̣pnłs & 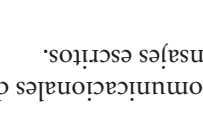 & 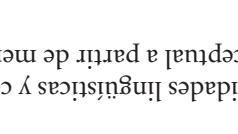 & 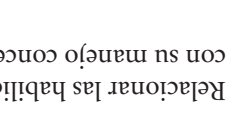 \\
\hline
\end{tabular}




\begin{tabular}{|c|c|c|c|c|c|c|}
\hline 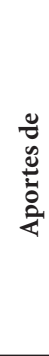 & 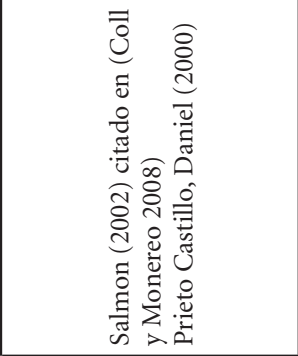 & 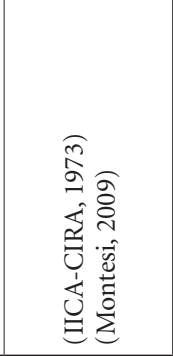 & \multicolumn{3}{|c|}{ 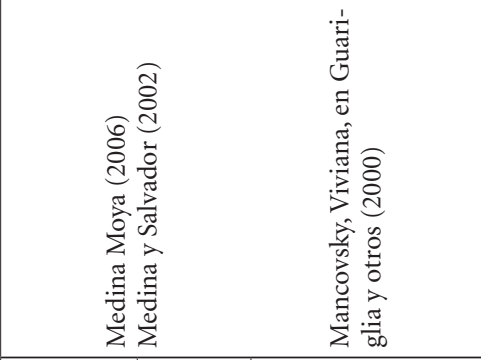 } & 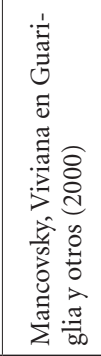 \\
\hline 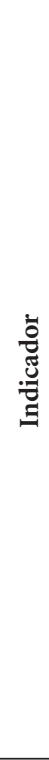 & 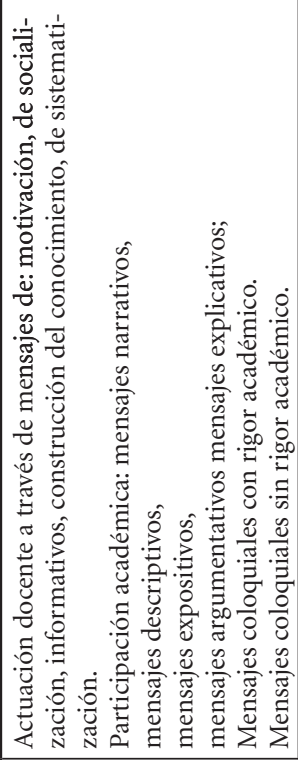 & 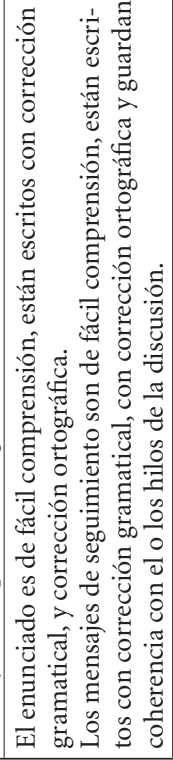 & 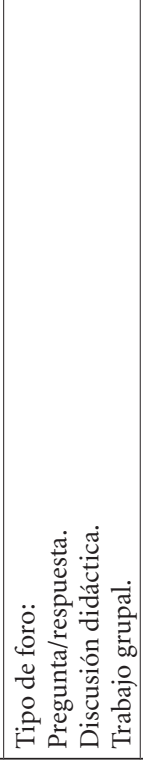 & 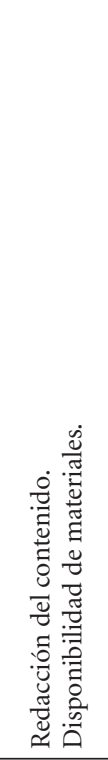 & 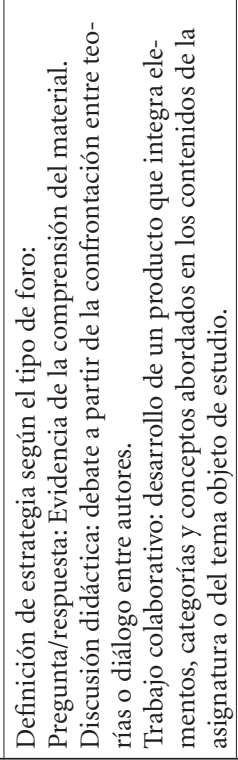 & 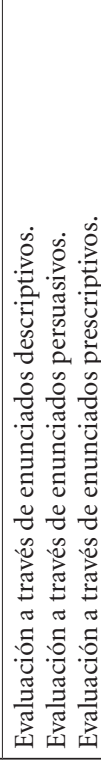 \\
\hline 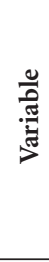 & 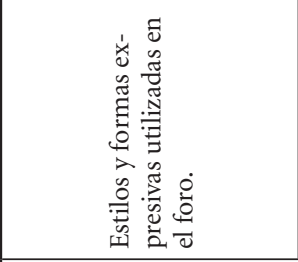 & 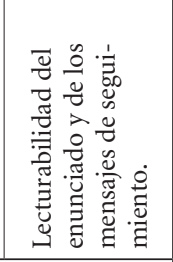 & 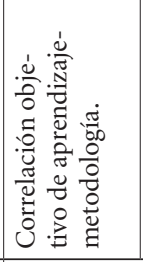 & 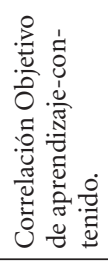 & 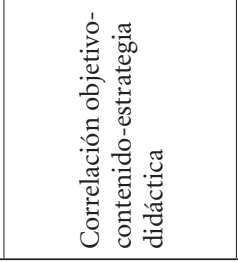 & 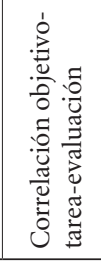 \\
\hline 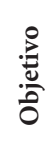 & 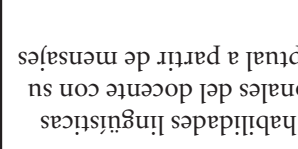 & 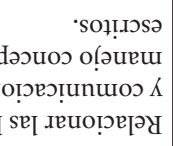 & 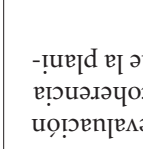 & 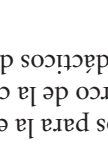 & 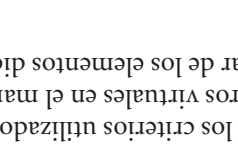 & 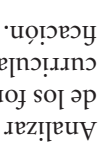 \\
\hline
\end{tabular}




\section{Unidades de análisis}

La investigación se ha realizado a partir del estudio de casos de foros de discusión desarrollados en las asignaturas de las maestrías mediadas por EVA, durante el período 2007-2010. De cada maestría se revisaron todas las asignaturas que aplicaron el foro como estrategia didáctica para el abordaje de alguno de los contenidos de estudio en su propuesta didáctico-metodológica.

\section{Nivel de análisis}

Las investigadoras hicieron la revisión de los:

- respaldos de las aulas virtuales de los programas de maestría de la muestra disponible en el entorno virtual de la UPS.

- instrumentos curriculares, de modo específico el plan microcurricular y la instrucción específica para la apertura del foro en cada una de las asignaturas de la muestra.

\section{Hallazgos y conclusiones}

\section{Sobre los tipos de foro planteados}

- Se ha observado que en el $82,35 \%$ de foros se llegan a trabajar las distintas fases de la etapa de construcción del conocimiento (inicio, intercambio, integración), que es la etapa intermedia de la construcción social del conocimiento. Solo un $11,76 \%$ genera la gestión del conocimiento, etapa final del aprendizaje colaborativo.

- El 75\% de los docentes muestra tendencia hacia la utilización del foro como respuesta a objetivos de aprendizaje de tipo cognitivo. En dos de cada tres casos la elaboración de la pregunta guía del foro y el tipo de mediación docente están encaminados al control de lectura de los materiales; el enunciado de la actividad define el tipo de foro pregunta-respuesta, y tiene como finalidad la evaluación del nivel de comprensión de elementos teórico/conceptuales del estudiante. Es decir, solo uno de cada tres foros se utiliza para la discusión didáctica, la estrategia didáctica se 
plantea a través de un enunciado que invita al diálogo o a la confrontación de posturas teóricas.

- Se constata, que la mayor parte de foros enfocados hacia lo teórico-conceptual son generales y en menor porcentaje se realizan foros grupales.

- En muchos casos el foro grupal se trabaja desde la dinámica de un foro general para la negociación e incluso el intercambio de argumentos. Existe una tendencia menor a utilizar el foro grupal como herramienta para la realización de trabajos en pequeños grupos desde un enfoque colaborativo con la finalidad de llegar a la integración de conocimientos, en los casos observados la propuesta se da como actividad en momentos intermedios o finales de la asignatura. Las estrategias suele complementarse, en algunos casos con la socialización de los productos como apoyo al aprendizaje de todos los participantes.

\section{Sobre los tipos de interactividad lograda}

- El diseño instruccional de los programas de estudios de posgrado en modalidad virtual de la UPS parte de la consideración de los conceptos de comunidad virtual de aprendizaje y entorno virtual de aprendizaje. De ahí que se consideren como principios básicos de la actuación didáctica la comunicación y la interacción como factores de construcción colectiva para la gestión del conocimiento.

- De la integración de elementos de análisis cualitativo y cuantitativo, se concluye que en el caso de foros de tipo pregunta-respuesta, se llega a la etapa de iniciación y en muy bajo porcentaje a la de intercambio/exploración, con niveles equitativos en lo participativo y cognitivo. La naturaleza de la comunicación se reconoce como alocución fundamentalmente y muy baja interlocución.

- Del análisis de los foros de discusión didáctica se observa una dinámica que avanza a la etapa de intercambio/exploración mayoritariamente, con un mediano porcentaje de foros en los que se llega a la etapa de negociación/integración y en casos específicos se llega a la de aplicación/resolu- 
ción, donde alternan niveles participativo, interactivo y cognitivo, expresados a través de la interlocución y la interacción, es decir, presencia de un diálogo medianamente fluido en el cual se puede percibir incluso la identificación de los estudiantes con una u otra línea de discusión emergente. Sin embargo, dada la condición asincrónica de la comunicación se observa que el diálogo no se procesa de manera lineal y que los niveles de participación alternan con los de interacción, provocando que existan mensajes de iniciación/exploración de algunos participantes del foro, cuando otros evidencian estar en una etapa de intercambio/exploración, e incluso en negociación/integración.

- En los foros destinados al trabajo grupal, se observan todas las fases consideradas en la etapa de construcción social del conocimiento, y se reduce el impacto de la asincronía de la comunicación durante el diálogo en relación al intercambio/exploración y a la negociación/interacción, siendo un factor a considerar el tiempo que transcurre entre la intervención de uno y otro estudiante. En este tipo de foro, se evidencia un nivel cognitivo final más o menos equitativo entre los participantes, aunque este resultado se ve condicionado directamente por cuán específico fue el enunciado de la actividad. En la mayor parte de casos no se llega al nivel metacognitivo donde el grupo reflexione sobre su propio aprendizaje. Así mismo, están presentes en todos los casos la comunicación a nivel de alocución, interlocución e interacción.

- En la mayoría de foros de la muestra no se da un proceso de cierre de la discusión por parte del docente, en pocos de estos casos son los estudiantes quienes proponen la sistematización. En su mayoría los foros no completan su proceso didáctico.

- La apertura de líneas de discusión surge desde el dominio personal de una categoría conceptual por parte de quien abre o propone la discusión. Se convierte en nudo crítico del tema inicial en cuanto sea un interés o necesidad cognitiva compartida por otros miembros del grupo. El nivel de participación e interacción - entendidos como cantidad y calidad de los mensajes de respuesta- se marca en función del reconocimiento académico del que goza ese participante, ya sea por el dominio de un tema 
o por sus habilidades comunicativas o por su capacidad de negociar significados en una discusión y es seguido regularmente por un colectivo de compañeros.

\section{Sobre el ciclo de aprendizaje colaborativo virtual y el manejo de cate- gorías conceptuales}

- Desde la prioridad que adquieren la comunicación asincrónica y escrita en los entornos virtuales de aprendizaje, se evidencia que el nivel de participación y de los mensajes llega solamente hasta las etapas de participación e interacción pues los mensajes son mayoritariamente de carácter informativo-narrativo. El aprendizaje social desde el e-learning se concreta fundamentalmente en las etapas de cognición y metacognición, para lo que se requiere un mayor dominio y aplicación de competencias comunicativas y habilidades lingüísticas del discurso escrito en tareas que lleven a la sistematización de saberes individuales y colectivos y que evidencien el dominio de conceptos clave y la discusión entre teorías.

\section{Sobre la mediación y el rol docente}

- Desde los foros revisados se concluye que una buena práctica de interacción del docente garantiza la gestión del conocimiento, pues no basta con hacer un planteamiento inicial de la discusión, aun cuando este sea específico, novedoso y claro, sino que es preciso moderar permanente el debate introduciendo nuevas categorías que promuevan la confrontación de teorías o el diálogo entre autores y sistematizar lo dicho sobre el objeto de estudio. Para llegar a esta etapa la estrategia metodológica sugerida tendría dos elementos posibles de aplicar; uno es la elaboración individual o colectiva de la sistematización de los aportes fundamentales debatidos sobre el objeto de estudio, y el otro, es la retroalimentación por parte del docente en donde recoja los elementos nucleares de la discusión y aborde aquellos menos discutidos o no tratados.

- A partir del análisis de los foros grupales y de los trabajos grupales o colaborativos se deduce que existe una mejor de gestión del conocimiento cuando se plantea la presencia cognitiva del estudiante hasta llegar a la 
etapa de aplicación o resolución. Solo desde la complementariedad de estos procesos se logra la presencia social y cognitiva -según Heri, Garrison y Anderson- que requiere el aprendizaje social en una comunidad virtual de aprendizaje.

\section{Sobre la coherencia curricular y los criterios de evaluación}

- La microplanificación de una asignatura precisa de coherencia curricular entre los elementos didácticos no personales de la planeación: objetivos, temas, procesos metodológicos, recursos, evaluación. Los foros deberían estar integrados al sistema de tareas de la asignatura de modo que sean orgánicos para la propuesta curricular, no es suficiente que sean una actividad para el abordaje de un tema específico o para el logro de un objetivo. Visto así, el foro delata su esencialidad y protagonismo en la gestión social de conocimiento.

- Los criterios de evaluación de foros y trabajos colaborativos planteados en la planificación microcurricular de cada asignatura pueden agruparse en cuatro grandes ámbitos de exigencia: competencias de actuación general, competencias cognitivas, competencias metacognitivas, competencias metodológicas colaborativas.

- Al momento de evaluar cuantitativamente la participación de un estudiante en el foro, es necesario explicitar la ponderación que tiene la nota respecto de cada uno de los criterios aplicados. Los criterios relativos a competencias de actuación general -presentes en la mayoría de casos estudiados-no son suficientes para evaluar el aprendizaje del estudiante, solo se está priorizando y reduciendo la evaluación al cumplimiento del "trabajo escolar" evidenciado a través de su asiduidad en el foro o presencia social.

- Los mensajes de seguimiento del foro que hace el docente son generalmente enunciados de tipo descriptivo sobre los contenidos conceptuales del objeto de estudio. Un bajo número de docentes utilizan enunciados persuasivos expresando satisfacción sobre la participación y producción 
en el foro o trabajo grupal. No se registran enunciados prescriptivos al momento de evaluar.

A partir de estos hallazgos y conclusiones se espera innovar el uso del foro virtual como estrategia de aprendizaje colaborativo y gestión colectiva del conocimiento, para los programas de posgrados en modalidad y metodología virtual de la institución en la que se desarrollan los programas caso de estudio de esta investigación.

\section{Bibliografía:}

BADIA, Antoni

2006 “Ayudar a aprender con tecnología en la educación superior». En: Antoni Badia (coord.). Enseñanza y aprendizaje con TIC en la educación superior [monográfico en línea]. Revista de Universidad y Sociedad del Conocimiento (RUSC). Vol. 3, n. ${ }^{\circ}$ 2. UOC. [Fecha de consulta: 06/09/2010]. http://www. uoc.edu/rusc/3/2/dt/esp/badia.pdf ISSN 1698-580X

BARBERÁ, Elena, T. Mauri y J. Onrubia

2008 Cómo valorar la calidad de la enseñanza basada en las TIC. Pautas e instrumentos de análisis. Colección Crítica y fundamentos. Barcelona: Editorial GRAO. Disponible en: http://books.google.com.ec. Consulta: 2010-09

BAYLON, Christian y X. Mignot

1996 La Comunicación. Ediciones Cátedra. Madrid.

COLL, César y C. Monereo (Comp.)

2008 Psicología de la educación virtual. Madrid: Ediciones MORATA. España.

CÓRICA, J. L.

2009 Diseño Curricular y Nuevas Generaciones. Buenos Aires: Editorial Virtual Argentina.

CASTORINA José Antonio y Dubrovsky, S.

2004 Psicología, cultura y educación. Perspectivas desde la obra de Vigotsky. Buenos Aires: Ed. Novedades Educativas. En: http://books.google.com.ec/ Consulta: 01-08-2011 
GUARIGLIA, Osvaldo y otros

2000 Reflexión ética en el campo de la educación y de la formación. Buenos Aires: Ediciones Novedades Educativa, Universidad Nacional de Buenos Aires.

IICA-CIRA.

1973 Curso sobre comunicación escrita. Bogotá: Instituto Interamericano de Ciencias Agrículas de la OEA.

LARA, Sonia.

Una estrategia eficaz para fomentar la cooperación. 99ESEN ${ }^{\circ} 1,2001$. Disponible en http://dspace.unav.es/dspace/bitstream/10171/7948/1/Notas4.pdf

LÉVY, P.

2004 Inteligencia colectiva. Washington: Organización Panamericana de la Salud.

LÓPEZ, Denisse e Ibis Álvarez

2009 "Promover la regulación del comportamiento en tareas de aprendizaje cooperativo en línea a través de la evaluación", publicado en la Revista Iberoamericana de Educación a Distancia, Vol. 14 Nº1, Junio 2011. Universidad Técnica Particular de Loja. Ecuador.

MARTÍNEZ, Francisco y M. Paz Prendes

2006 “Actividades individuales versus actividades colaborativas". en J. Cabero, \& P. Román, E-actividades. Un referente básico para la formación en Internet (pág. 183). Sevilla: Editorial MAD En: http://books.google.com.ec.

MANCOVSKY, V.

2000 "Hacia una mirada reveladora de los proceso que recubren la interacción pedagógica”. en O. e. Guariglia, La reflexión ética en el campo de la educación y la formación (págs. 85-114). Buenos Aires: Ediciones Novedades Educativas.

MEDINA, Antonio y F. Salvador

2002 Didáctica general. España: Pearson.

MEDINA, José Luis

2006 La profesión docente y la construcción del conocimiento profesional. Argentina: Editorial Lumen. 


\section{MONTESI, M.}

2009 Aproximación al documento textual desde la perspectiva de los estudios sobre el discurso. Recuperado el 04 de 07 de 2011, de Revista Española de Documentación Científica: En: http://redc.revistas.csic.es/index.php/redc/ article/view/516/574 Consulta: 04-07-2011

PERRENOUD, Philippe

2007 Diez nuevas competencias para enseñar. Madrid: Ediciones Graó.

PRIETO, Daniel

2000 Análisis de mensajes. Quito: Ediciones CIESPAL.

PUENTE-PALACIOS, Katia y A. Severino

2010 "Cogniciones compartidas: una revisión sobre memoria transaccional". Revista Alteridad No 8. Quito: Universidad Politécnica Salesiana/Abya-Yala.

RODRÍGUEZ-PONCE, Emilio y L. Pedraja-Rejas

2007 Efectos e implicaciones de las decisiones estratégicas en las instituciones universitarias. Revista digital Interciencia, septiembre 2007, VOL. 32 No 9 , ISSN 0378-1844. Disponible en http://www.interciencia.org. Revisado 2308-2011.

SALMERÓN, Honorio, S. Rodríguez y C. Gutiérrez

2010 "Metodologías que optimizan la comunicación en entornos de aprendizaje virtual", en Comunicar, 163-171.

SILVA, Juan

2011 Diseño y moderación en entornos virtuales de aprendizaje. Barcelona: Editorial UOC. Barcelona. 